\title{
Characteristic Human Scent Compounds Trapped on Natural and Synthetic Fabrics as analyzed by SPME-GC/MS
}

\section{PA Prada, AM Curran and KG Furton*}

Florida International University, Department of Chemistry \& Biochemistry, Modesto Maidique Campus, Miami, FL

*Corresponding author: KG Furton, Florida International University, College of Arts and Sciences, ECS 450, 11200 SW 8th Street, Miami, FL 33199, E-mail: furtonk@fiu.edu

Citation: PA Prada et al. (2014) Characteristic Human Scent Compounds Trapped on Natural and Synthetic Fabrics as analyzed by SPME-GC/MS. J Forensic Sci Criminol 1: S101. doi: 10.15744/2348-9804.1.S101

Received Date: December 10, 2013 Accepted Date: May 08, 2014 Published Date: May 12, 2014

\begin{abstract}
The collection of human odor volatiles is of interest to forensic applications as a path to investigate canine scent discriminations in legal investigations. A study using a selected array of previously identified human odor compounds has been conducted to determine the retention and release capabilities of five (5) natural and synthetic fabric types, cotton (mercerized fabric and gauze matrix), polyester, rayon and wool. A direct spike approach as well the use of a dynamic airflow device were the two approaches used for the collection of the selected volatile organic mix. The direct spike experiment showed how natural, cellulosic fibers such as viscose rayon showed an enhanced ability to release a reproducible volatile odor profile. Rayon demonstrated to be the fabric type with the highest recovered scent mass amounts, followed by wool and polyester. As was expected cotton showed the lowest recovered amounts, possibly due to its complex fiber morphology which enhances the possibility of chemicals to be retained in higher rates within the structure of the cotton fiber. Samples collected on the same fabric substrate showed a reproducible odor profile as measured via hierarchical clustering which corroborates previous live human odor studies and which can be pivotal in forensic biometric measurements. The introduction of an airflow variable to volatile collection decreased the amounts recovered for all fiber types. The reproducibility for each fabric type between replicate sampling was also reduced and a statistical significant difference $(\mathrm{P}<0.001)$ was observed in the interaction between airflow speed and fabric type. The cotton fabric was the material which showed enhance collection at the low airflow speed as observed by the recovered mass amount. In conclusion, these findings do indicate that chemical retention is strongly affected by fiber type and outside environmental variables such as airflow, which can alter the odor profile of a collected scent sample.
\end{abstract}

Keywords: Human odor; Volatile organic compounds (VOCs); Fabric substrates; Dynamic airflow

\section{Introduction}

Human scent is a form of trace evidence that can provide associations between suspects, victims and crime scenes. With the aid of a well-trained canine, human scent can allow an investigative team to follow a suspect from the location of a crime, determine the direction of travel of an assailant, or even identify a suspect in a formal scent line-up procedure. From a forensic standpoint, the definition of human hand odor volatiles has been a pivotal area of research as this body region comes into contact with crime scene objects with high frequency. Thus, the need to elucidate human odor via instrumental means has been at the forefront of enhancing this forensic tool.

In general, human odor can be described as a combination of volatile compounds released from the body that are directly affected by genetics, environment and physiological secretions. It is known that the epidermis (outer) layer of the skin constantly sheds epithelial cells into the environment, which further contribute to an individual's odor profile in the form of sweat, oils and other glandular secretions [1]. Contributing to unique personal characteristics of human skin odor are factors such as moisture, temperature, bacterial flora and $\mathrm{pH}$ on an individual's skin surface [2-4] Interactions between bacterial flora and textiles have been studied in the understanding of odor generation patterns [5]. Morphological characteristics of fiber structures such as weave and fiber tightness have also been found to play key roles in the adherence of bacteria and thus in the generated body odor [6-10].

Even though axillary odor produces the majority of human mal-odor constituents, it is not the only source of an individual's wholebody odor signature. Thus, various instrumental evaluations have focused on other body regions such as the hands and forearms for the evaluation of skin volatiles that may contribute to the definition of general whole-body scent. Information gathered from studying an individual's VOC profile has many applications, including: the diagnosis of disease [11], cosmetic industry [12], mosquito attractant detection [13-16] as well as for biometric purposes in a forensic context [17]. Yet these studies have focused on elucidating the definition of body odor in different perspectives, the effect of fabric properties on odor collection and retention has not been fully exploited. 
In the context of forensic science for example, odors from hands can serve as individualized characteristics for scent discrimination investigations. Thus, evaluation of an optimal sorbent material for standardizing forensic protocols is key in this particular application. Other studies have focused on chemical retention on distinctive fiber types. It has been shown that adsorption is heavily influenced by chemical structure and hydrophobic interactions. The high polarity of cellulosic fibers for example, can act as a driving force for the penetration of chemicals into the pores and voids of the fibers. Thus, the physicochemical nature of fibers (i.e. micropores, fibrils, surface and secondary wall) play a key role in the ultimate retention and distribution of chemicals. Additionally, chemicals with low vapor pressures are retained on fabrics to a larger extent than chemicals with higher vapor pressures [18-20].

The Furton research laboratory has pioneered the instrumental analysis of human hand odor samples using gauze pads as the collection medium. These studies have not only identified key volatile organic compounds for odor discrimination [21], but also statistical techniques to corroborate their usefulness as volatile "barcode" markers for identification purposes [22]. Furthermore, an active research quest in the laboratory has been the development of optimal human odor collection procedures, with an emphasis in the types of collection mediums. A study using both female and male subjects compared hand odor volatiles collected on various textiles which highlighted how cotton materials released the most abundant scent mass amounts and how the chemical odor profile is directly dependent on the textile used [23]. Due to the complex interaction between skin microflora and glandular secretions, together with the regulatory action of the specific body regions, the transfer of odor to the particular fabric is not easily controlled in a laboratory setting when sampling human subjects. Furthermore, the varying compositions and quantities of these microbial colonies on the surface of the skin yield to highly variable amounts of intensity and quality of the generated body odor.

Thus, in an effort to elucidate the interactions between specific human odor compounds in relation to textile types, pure compounds have been used to explore this collection variable in a generated odor profile. In a study conducted by Hudson and Furton [24], three commonly used gauze mediums employed by national and international law enforcement agencies were evaluated for their retention capabilities using previously identified human scent compounds. The results from this study showed how cotton blend gauze mediums (cotton, rayon and polyester) released greater number of polar compounds than $100 \%$ cotton gauze mediums. In a subsequent study [25], only 5 standard compounds (6-methyl-5-hepten-2-one, furfural, tetradecane, furfuryl alcohol and dimethyl ester hexanedioic acid) were used for collection procedures using pure cotton, polyester, rayon fabrics and also cotton-blended gauze pads. In this analysis, a dynamic airflow device was employed for collection as opposed to a direct spike of compound, where again cotton-containing materials showed the highest release of target compounds.

The objectives of this study are to further evaluate the retention and release capabilities of five textile types employing a human scent compound standard mixture to reduce the influence of participant variation on the collected sample. Fabrics of both natural and synthetic origin were tested using both direct contact and a dynamic airflow system in an effort to understand the retention and release of previously identified human odor compounds on various fabric substrates.

\section{Material/ Methods}

The four different types of textile materials used in this study included: bleached desized mercerized cotton print cloth, spun viscose challis, spun polyester type 54 and 100\% wool flannel (Test Fabrics, West Pittston, PA). The absorbent gauze material evaluated was DUKAL brand 100\% cotton, sterile 2x2", 8ply, gauze sponges (Dukal Corporation, Hauppauge, NY). All experiments used a $2 \times 2$ " piece of textile square for chemical collection procedures. The SPME fibers used for the headspace extraction of these samples were Divinylbenzene/Carboxen on Polydimethylsiloxane (gray) 50/30 $\mu \mathrm{m}$ (Supelco, Bellefonte, PA). Chemicals used for the volatile organic mix used in all experiments were obtained from Sigma Aldrich, St. Louis, MO, USA and included: nonanal, 95\%, Batch \#05223DC, decanal, Batch \#086K1467, 2-furancarboxaldehyde, 99\%, Batch \#03920KB, dodecane, 99\%, Batch \#00654LC, tetradecane, 99\%, Batch \#13401LZ, 6-methyl-5-hepten-2-one, 99\%, Batch \#06723DU, 6,10-dimethyl-5,9-undecadien2-one, 97\%, Batch \#03906JC, 2-furanmethanol, 99\%, Batch \#07624KC, octanoic acid methyl ester, 99\%, Batch \#260673, hexanedioic acid dimethyl ester, 99\%, Batch \#186252, octanoic acid, 98\%, Batch \#03907, phenol, 90\%, Batch \#981343.

\section{Pretreatment of Fabric Substrates}

All samples were placed inside $10 \mathrm{~mL}$ screw-top vial (Supelco, Bellefonte, PA). All glass vials were rinsed with acetone and baked at $105^{\circ} \mathrm{C}$ for 48 hours to remove any volatile organic compound (VOC) remnants from vials prior to actual sample collection. Prior to chemical sampling, the fabrics were analytically cleaned, as it has been shown from previous studies that sterile does not equate to a volatile free material [26]. Pre-treatment of all fabric materials consisted of a direct spike with one milliliter of HPLC grade methanol (Fisher Scientific, Pittsburgh, PA, USA) followed by heating at a temperature of $105{ }^{\circ} \mathrm{C}$ for one hour in an oven (Fisher Scientific Isotemp Oven Model 655G) to eliminate any volatile organic compounds present on each substrate. Each material was then analyzed by SPME-GC/MS (same method as described below) to establish the background levels prior to chemical spiking.

\section{SPME-GC/MS Method}

For the chromatographic separation a Hewlett Packard 6890 Series GC system, fitted with an HP5-MS 30 meter, $0.25 \mu$ m, and 0.25 mm capillary column was used. The GC oven temperature was programmed as follows: $5 \mathrm{~min}$ at $40^{\circ} \mathrm{C}$, then heating at $10^{\circ} \mathrm{C} / \mathrm{min}$ up to $300^{\circ} \mathrm{C}$ and held for $2 \mathrm{~min}$, for a total analysis time of $33 \mathrm{~min}$. Helium was the carrier gas at a flow rate of $1 \mathrm{~mL} / \mathrm{min}$ at an average velocity of $37 \mathrm{~cm} / \mathrm{sec}$. 
The injection was conducted in the splitless mode. The inlet had an initial temperature of $250{ }^{\circ} \mathrm{C}$ with a pressure of 7.00 psi. The purge flow was $16.4 \mathrm{~mL} / \mathrm{min}$ for a period of 2.00 minutes. The total flow was $20.1 \mathrm{~mL} / \mathrm{min}$. The mass spectrometer used was an $\mathrm{HP}$ 5973 MSD with a quadrupole mass analyzer which was operated in electron ionization (EI) mode and scanned over a mass range of $\mathrm{m} / \mathrm{z} 45-550$ in full scan mode. Compounds were identified by retention time match with reference standards and by using the NIST 98 mass spectral reference library search. The amount of each compound extracted by the SPME fiber was determined using external standard calibration.

\section{Scanning Electron Microscope (SEM) Imaging of Textile Surface}

The scanning electron microscope (SEM) was a JEOL JSM-5900LV ran under low vacuum mode with an acceleration voltage of $15 \mathrm{kV}$ and a pressure of $21 \mathrm{~Pa}$ for both the $\mathrm{x} 65$ and $\mathrm{x} 400$ amplification analyses. The differences in fiber composition were evaluated by performing scanning electron microscope imaging (SEM) to further identify possible weaving pattern and fiber structure distinctions which may affect the actual collection, retention and dissipation of human scent compounds. The bleached desized mercerized cotton print cloth, spun viscose challis, spun polyester type 54, 100\% wool flannel and the $100 \%$ cotton, sterile $2 \times 2$ ", 8 ply, Dukal gauze sponge pads were cut into small square pieces. The pieces of material were not coated for SEM imaging and were simply mounted on an aluminum stub with carbon adhesive. Pieces were arranged to fit eight pieces of material per stub. The JEOL JSM-5900LV was then used to image the surface of each sorbent material at both 65X and $400 \mathrm{X}$ magnifications.

\section{Direct Fabric Spike of Volatile Organic Compound Mixture}

To evaluate the differences in trapping and release capabilities of the various fabric materials, a 40 ppm standard mixture of representative human scent compounds was utilized. Table 1 lists the compounds that were used in this mixture based on the reported frequency of occurrence in odor samples from previous literature [23]. High frequency occurring compounds include 2-furanmethanol, phenol, 2-furancarboxaldehyde, nonanal, decanal and dimethyl ester hexanedioic acid. Medium frequency occurring compounds include dodecane, 6-methyl-5-hepten-2-one and 6,10-dimethyl-5,9-undecadien-2-one. The low frequency occurring compounds in the mixture are tetradecane and methyl ester octanoic acid. Octanoic acid was also incorporated in the mixture so as to include a range of functional groups and has also been reported in the literature as human emanating compound. The experiment was conducted in triplicate by spiking $10 \mu \mathrm{L}$ of the $40 \mathrm{ppm}$ volatile organic compound mixture in dichloromethane onto each sorbent material (bleached desized mercerized cotton print cloth, spun viscose challis, spun polyester type 54, 100\% wool flannel and Dukal 100\% cotton gauze pad). After spiking, each material was immediately sealed in the 10-ml glass vials. Positive controls were also made in triplicate by spiking $10 \mu \mathrm{L}$ of the $40 \mathrm{ppm}$ standard mixture in an empty $10 \mathrm{~mL}$ glass vial. Both the spiked materials and the positive controls were allowed to equilibrate for 24 hours prior to analysis. The SPME fiber sampled the headspace for a period of 21 hours prior to GC/MS analysis. A 6 point calibration curve (5-80 ppm) was used for external standard calibration. The amount of recovered VOCs for each sorbent material was consequently analyzed and compared.

\begin{tabular}{|l|l|l|l|l|}
\hline \multicolumn{1}{|c|}{ Compound Name } & Retention Time (min) & Molecular Weight $(\mathrm{g})$ & Vapor Pressure (Torr) & Functional Group \\
\hline 2-furanmethanol & 7.21 & 98.1 & $6.098 \mathrm{E}-01$ & Alcohol \\
\hline Phenol & 10.33 & 94.1 & $2.199 \mathrm{E}-02$ & Alcohol \\
\hline 2-furancarboxaldehyde & 6.51 & 96.1 & $1.870 \mathrm{E}-01$ & Aldehyde \\
\hline Nonanal & 12.52 & 142.2 & $3.330 \mathrm{E}-02$ & Aldehyde \\
\hline Decanal & 14.16 & 156.3 & $9.570 \mathrm{E}-03$ & Aldehyde \\
\hline Dodecane & 14.04 & 170.3 & $7.203 \mathrm{E}-03$ & Aliphatic \\
\hline Tetradecane & 16.86 & 198.4 & $5.354 \mathrm{E}-04$ & Aliphatic \\
\hline 6-methyl-5-hepten-2-one & 10.32 & 126.2 & $7.501 \mathrm{E}-01$ & Ketone \\
\hline 6,10-dimethyl-5,9-undecien-2-one & 17.58 & 194.3 & 2.427E-02 & Ketone \\
\hline Octanoic acid, methyl ester & 12.84 & 158.2 & 174.2 & $3.7598 \mathrm{E}-05$ \\
\hline Hexanedioic acid, dimethyl ester & 14.70 & 144.2 & Fatty acid methyl ester \\
\hline Octanoic acid & 14.22 & Fatty acid methyl ester \\
\hline
\end{tabular}

Table 1: Human Scent Compound Mixture Composition

\section{Dynamic Air Sampling of Representative Standard Mixture}

The dynamic air sampling study was conducted utilizing the STU-100 (Bill Tolhurst Enterprises, Haw River, NC, USA), which includes a portable case with a 12 volt battery and the control for the vacuum pump airflow. The STU-100 has nine different airflow speeds ranging from 1 to 9 and a modified inlet for holding a sterile gauze pad $(12.5 \mathrm{~cm} \mathrm{x} 23.0 \mathrm{~cm})$. This gauze pad is used as the trapping medium to collect volatile components as the STU-100 pulls air through the sorbent at the running airflow rate [27]. The STU-100 model used in this study was modified from the original Teflon-coated hood version and has been described elsewhere [23].

Using the 40 ppm standard mixture of representative human scent compounds (see Table 1), the four different textile types and gauze material mentioned above were evaluated at the low (1), medium (5) and high (9) airflow settings of the STU device. 
To monitor the airflow volume passing through each material, an anemometer was placed directly above the circular opening of the metal plate, where 10 consecutive readings were taken for one minute at the low, medium and high airflow settings. The experiment was conducted at indoor laboratory conditions $\left(22.7^{\circ} \mathrm{C} / 57.7 \%\right.$ relative humidity). STU hood and metal plate placed overnight in oven at $105^{\circ} \mathrm{C}$ prior to any sampling. In between samples, plate and hood were cleaned with alcohol pads. A blank sample was taken prior to addition of the spike mixture. This was performed by running the device at each evaluated airflow above an empty Petri dish. For the standard mixture sampling, three consecutive samples were taken of the standard mixture with only an allotted break for cleaning the device with alcohol pads. Three different Petri dishes were used and each had approximately $30 \mathrm{~mL}$ of the 40 ppm VOC standard mixture. Samples were allowed to equilibrate for 24 hours prior to headspace SPME extraction for 21 hours.

\section{Statistical Analysis}

The source of variation between fabric samples was evaluated by using both one and two-way ANOVA analysis. One way ANOVA allowed the comparison between the average masses of all fabric types and used to determine if there were significant differences in the recovered mass from each fabric at a 95\% confidence interval for the direct spike study. In order to further analyze the pattern of difference between the means, the ANOVA was followed by a Fisher's least significance difference post hoc test. ANOVA was used to verify if the variation was due to random error in measurements or to a single controlled factor, such as fabric type. Furthermore, in the dynamic airflow study a 2-way ANOVA was used to examine the influence of both fabric type and airflow speed on chemical collection. Hence, it tested the interaction effect between both variables.

For this study, hierarchical clustering was used to determine the relatedness of each sample compound profile as a function of fabric type. A dendogram was constructed with IBM SPSS statistical software version 21 (IBM Corporation).

\section{Results and Discussion}

SEM Imaging

The differences in fiber composition were initially evaluated by performing scanning electron microscope imaging (SEM) to identify possible weaving pattern and fiber structure distinctions which may affect the actual collection, retention and dissipation of target human scent compounds. As can be observed from Figure 1, the cotton fabric exhibits a natural texture as seen by the fiber twists. The Dacron polyester and rayon fabric exhibit similar weaving patterns, however polyester fibers exhibit a narrower, smoother finish when compared to the viscose rayon fibers. Cotton, polyester and rayon can be said to have a tight woven structure, which may directly affect the collection of human scent by trapping the compounds in their weave and perhaps retaining odor for a more prolonged time. The wool woven structure is less structurally organized and is more like a mesh of fibers which when placed under higher magnification portray the complex morphological structure of what seems to be animal hair structure properties. The Dukal sterile pad illustrates a loose woven pattern when compared to all the other tested sorbent materials, thereby providing the most differing characteristics of weaving properties.

a) Bleached Mercerized Print Cloth $\mathrm{X} 65$

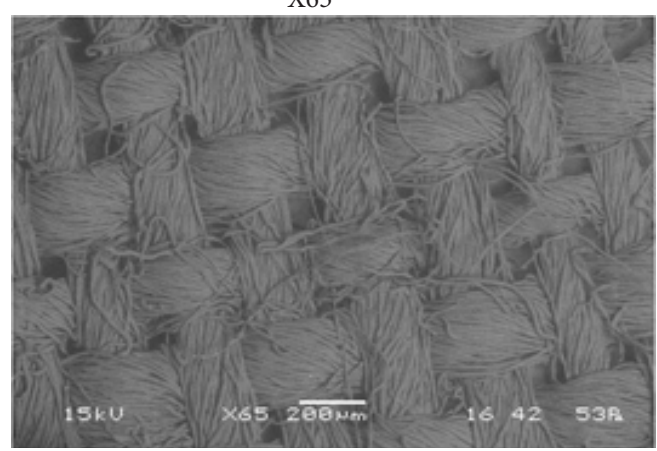

b)Dacron Polyester Fabric

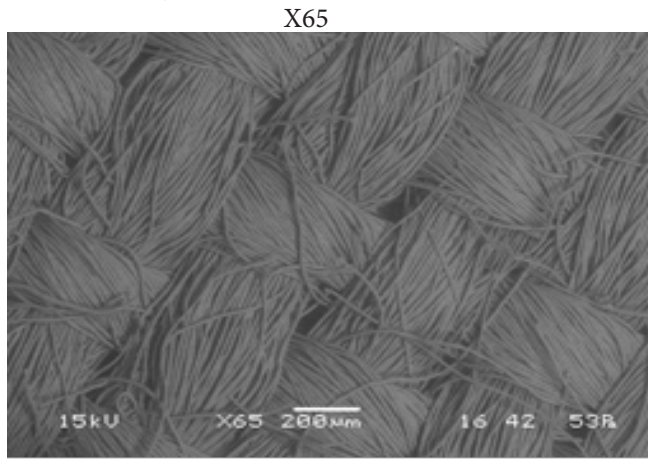

$\mathrm{X} 400$

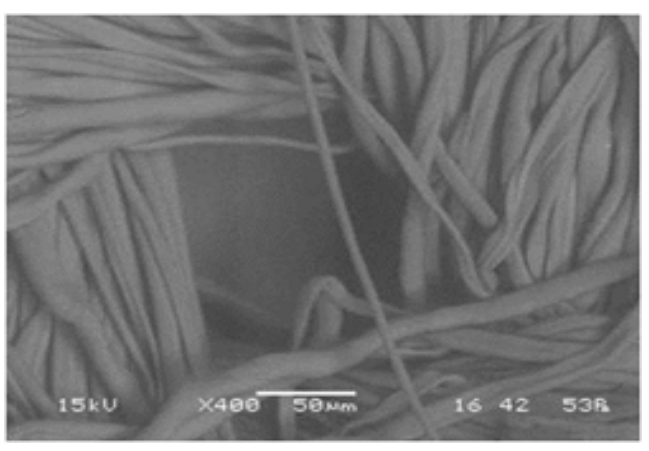

$\mathrm{X} 400$

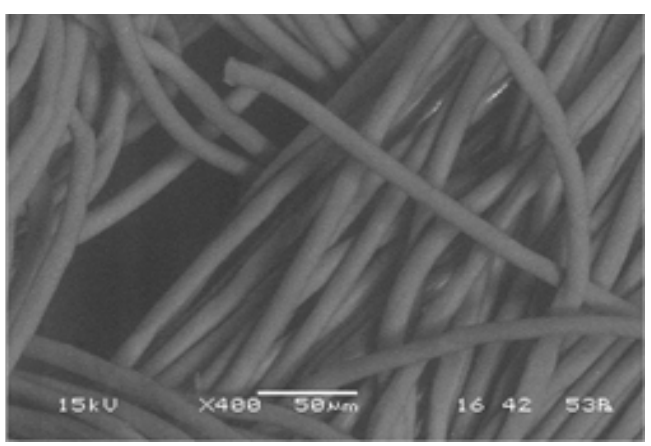


c) Viscose Rayon Fabric

X65

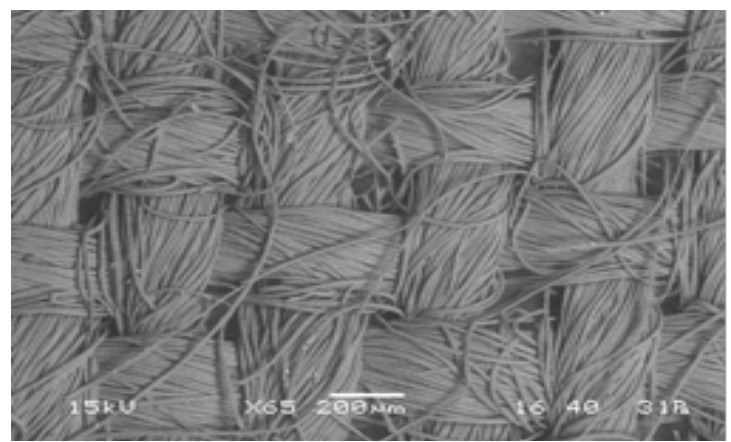

d) Wool Flannel

X65

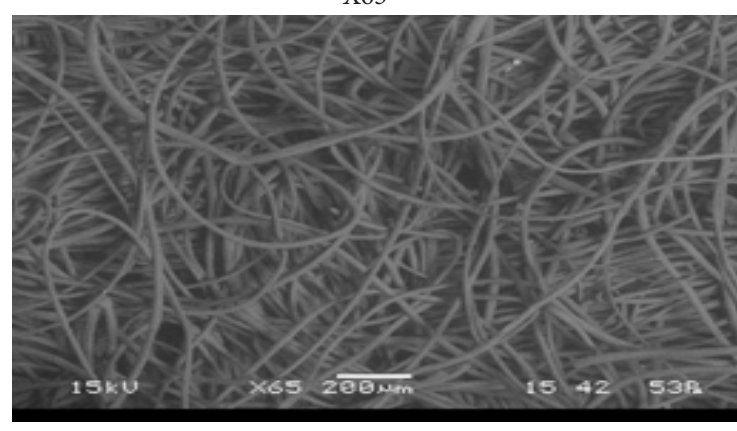

e) Dukal Sterile Gauze Pad

$\mathrm{X} 65$

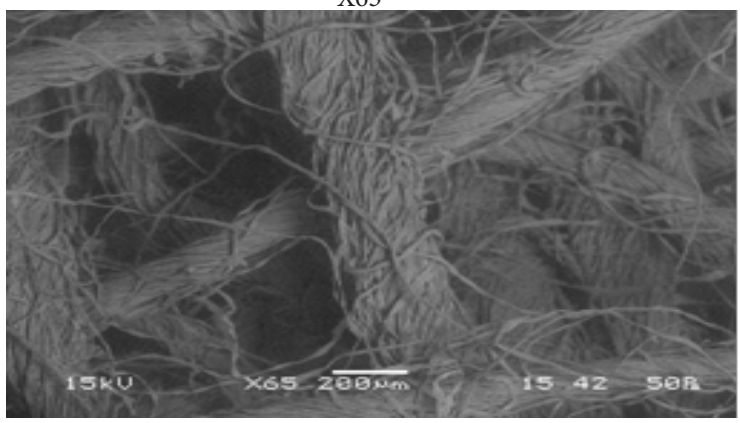

$\mathrm{X} 400$

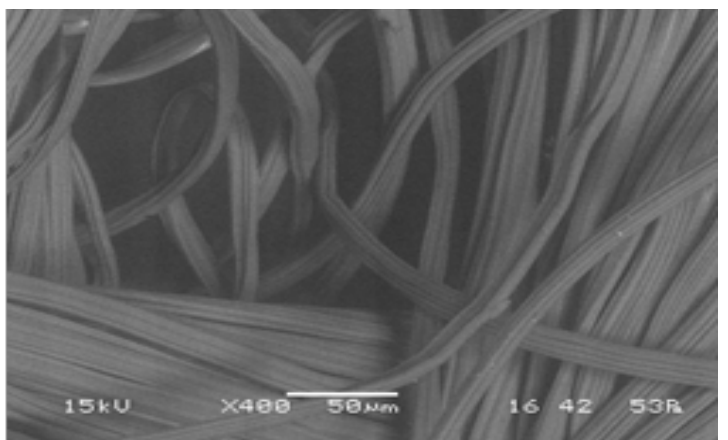

$\mathrm{X} 400$

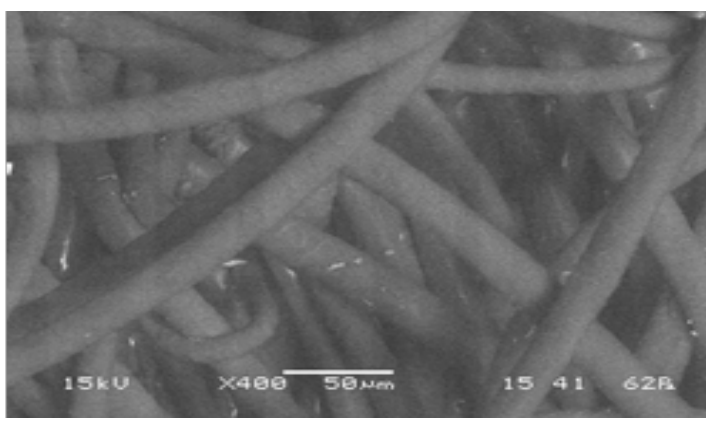

$\mathrm{X} 400$

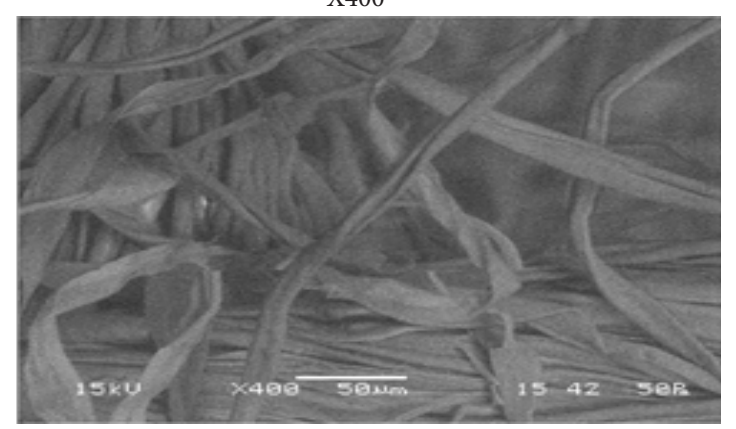

Figure 1: SEM Images of Fabric Substrates

Wool fibers are almost round in cross section and consist of cortical cells which run parallel to the fiber as well as the scales that cover the surface of this type of fiber. As shown in Figure 1-d, the scales under the 400x magnification give distinct frictional properties to the fiber which allows these fibers to easily slide past one another. The fiber morphology also depends on the breed of sheep and may exhibit differences not only from the scales, but also from the natural crimp.

\section{Direct Chemical Spiking on Fabric Materials}

From previous studies by the authors [23], human scent odor profiles are distinctive based on the textile selected for collection purposes. These observed differences showed the importance of fabric selection in providing a reproducible sample for differentiation purposes. Thus, the use of a human scent standard mixture allowed the comparison of fabric types without the variances encountered when using human subjects to further understand these differences in retention and consequent release of target odor compounds.

Of the total amount spiked in the $10 \mathrm{~mL}$ glass vial (positive control), an average of $1288 \mathrm{ng}$ was recovered using SPME-GC/MS. The total mass of VOCs spiked into the positive control was $4800 \mathrm{ng}$, indicating a recovery of approximately a $26.8 \%$ of the total mass by the headspace extraction. From the Dukal ${ }^{\oplus}$ cotton gauze an average of $108 \mathrm{ng}$ of compounds was recovered; cotton fabric followed with $261 \mathrm{ng}$ of VOC mass, polyester reported $398 \mathrm{ng}$ of mass, wool a mass of $605 \mathrm{ng}$, while the rayon fabric had the highest recovered VOC mass with an average of 664 ng (Figure 2).

The use of a standard mixture was a non-biased manner in which to monitor the amount and types of volatiles being collected on each sorbent medium without the variability seen when sampling actual human subjects. Rayon, a cellulosic regenerated fiber type, proved to provide with the highest mass recovery. 


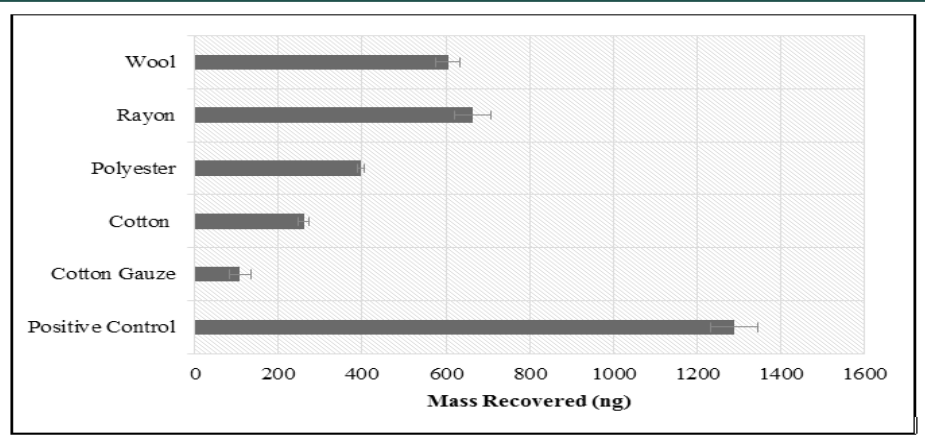

Figure 2: Average VOC Mass Recovered from Control and Sorbent Materials

Furthermore, even though two cotton type of materials were evaluated (fabric and gauze), from the results obtained it seems that the fabric is able to trap and release a greater quantity of VOCs in the headspace. The low amount seen with the gauze material may be attributed to the loose weave pattern observed that may have prevented the actual VOCs to be trapped originally or perhaps a greater affinity of the spiked VOCs with the polar cellulosic backbone of the material. Statistically, a one way ANOVA was performed to compare the mean of the recovered mass amounts from each material type. At a 95\% confidence level, the calculated $F$ value was greater than the critical $F$ value thus showing a significant difference from the recovered masses. A least significant difference (LSD) test was consequently done upon the rejection of the null hypothesis. Comparisons of the difference between the means of each material type were then compared to the LSD value. The comparisons to the calculated LSD highlighted that the amount of VOC mass recovered by the rayon and wool were not significantly different to each other, however, the amounts recovered by the cotton fabric, cotton gauze and polyester was significantly different to the other material types.

The standard mixture in Table 1 was comprised of six major functional groups including aldehydes, aliphatics, acid esters, acids, ketones and alcohols. Two compounds, 2-furanmethanol and 2-furancarboxaldehyde, were not detected in any of the tested fabric types. This could either be a result from a lack of retention by the SPME fiber at the time of extraction, or a high affinity of the compound toward the textile's fiber structure. The aliphatic hydrocarbon, being present in all materials in great abundance exhibited the highest recovery (Figure 3). The wool retained a significant mass of ketones, acid esters and aliphatic hydrocarbons. As acids are polar compounds, they have a higher affinity for polar backbones. This is important to note when conducting human odor sampling, as the choice of collection material may inadvertently cause the presence/absence of certain volatile compounds due to interactions with the fiber chemistry. In turn, this may cause differences in the odor profiles obtained for the same individual when using a variety of collection materials. This could represent an instrumental challenge for distinguishing odor profiles of the same person when changing the fabric type.

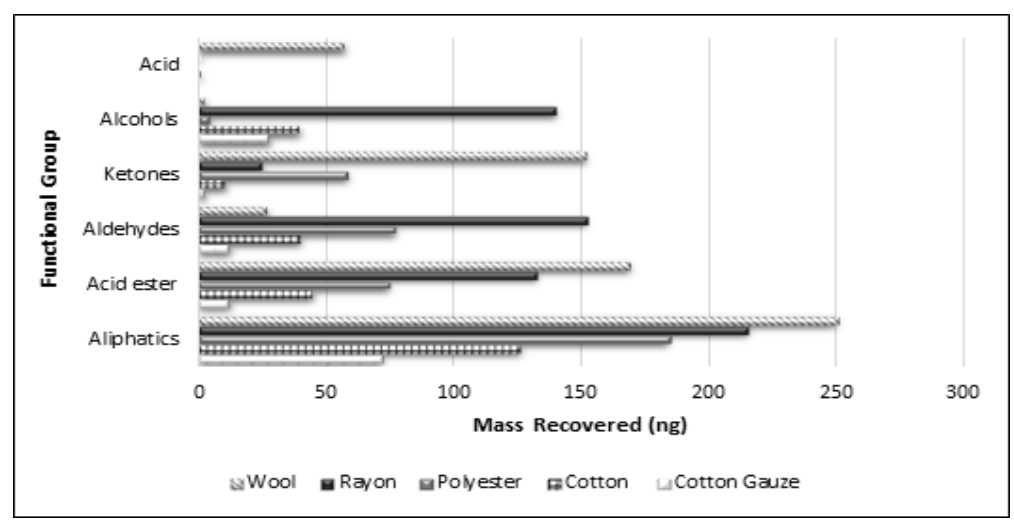

Figure 3: Functional Classes of Compounds Recovered from Chemical Spike across

A hierarchical clustering analysis was performed to compare the similarity of the volatile odor profile in relation to fabric type (see Figure 4). In general, all 5 fabric types clustered with samples of the same group. The most similar samples were the clusters of the cotton and polyester fabrics. Furthermore, there was a clear clustering of the cotton fabric types, gauze and pure fabric, thereby showing the similarity of odor profile. This confirms that samples collected on the same fabric are more similar to each other than to other fabric types. Hence, the importance of using a homogeneous fabric type when collecting live human odor.

\section{Fabric Sampling with a Dynamic Airflow System}

Chemical retention and release on the various fabric types was also evaluated using a dynamic airflow device. In forensic investigations, it is a common routine to collect human scent evidence articles using the STU-100. Thus, part of the study entailed an evaluation of the same five fabric types with the introduction of airflow as part of the chemical collection process. In this manner, our work simulates evidence collection, as this method uses sorbent materials to capture volatiles while in close proximity to an article of evidence. Airflow volume measurements were conducted prior to any chemical collection with/without any fabric loaded on the surface at the low, medium and high airflow speeds. The results are summarized in Figure 5. 


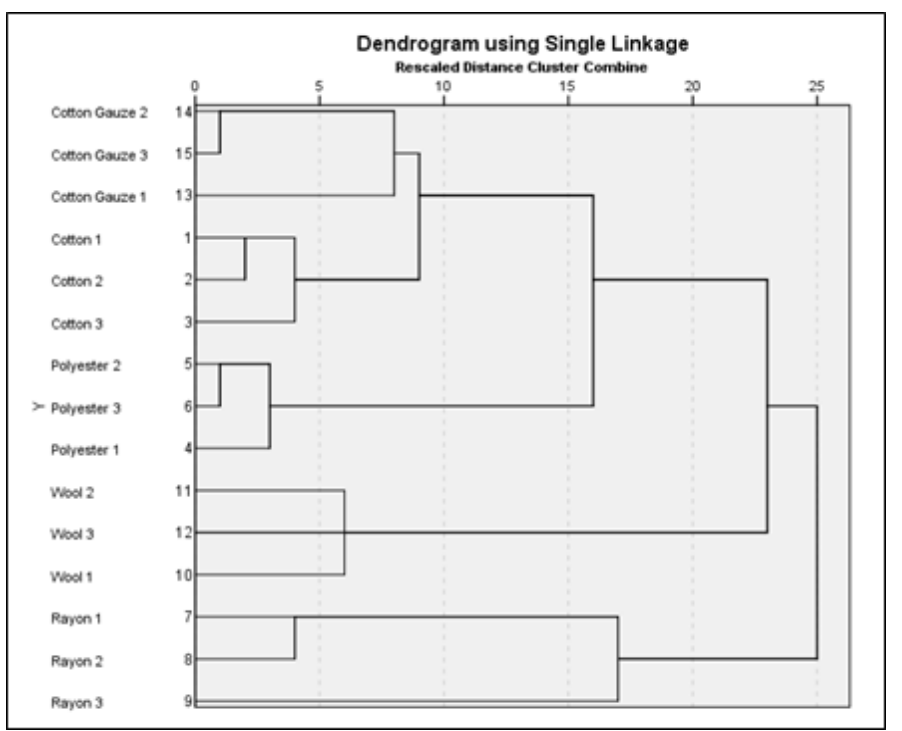

Figure 4: Cluster Analysis Dendogram of Direct Spiking Experiment on Different Fabric Substrates

As can be observed, without any fabric loaded the airflow volume is the largest (as was expected) reaching approximately $538 \mathrm{~L} /$ min at the highest speed. Dukal cotton gauze is the material with the highest reported airflow volume rates at all speeds evaluated followed closely by the pure cotton fabric. This was to be expected, as from the SEM imaging results the Dukal gauze material had the most open weave pattern $(1.5 \mathrm{~mm}$ between wefts and wraps). Thus, cotton materials presented the largest airflow volume when conducting non-contact sampling. The polyester fabric had the lowest airflow volume rates at all airflow settings which could explain the low mass amounts observed with chemical standard samples as perhaps not enough air is passed to collect VOCs onto material surface.

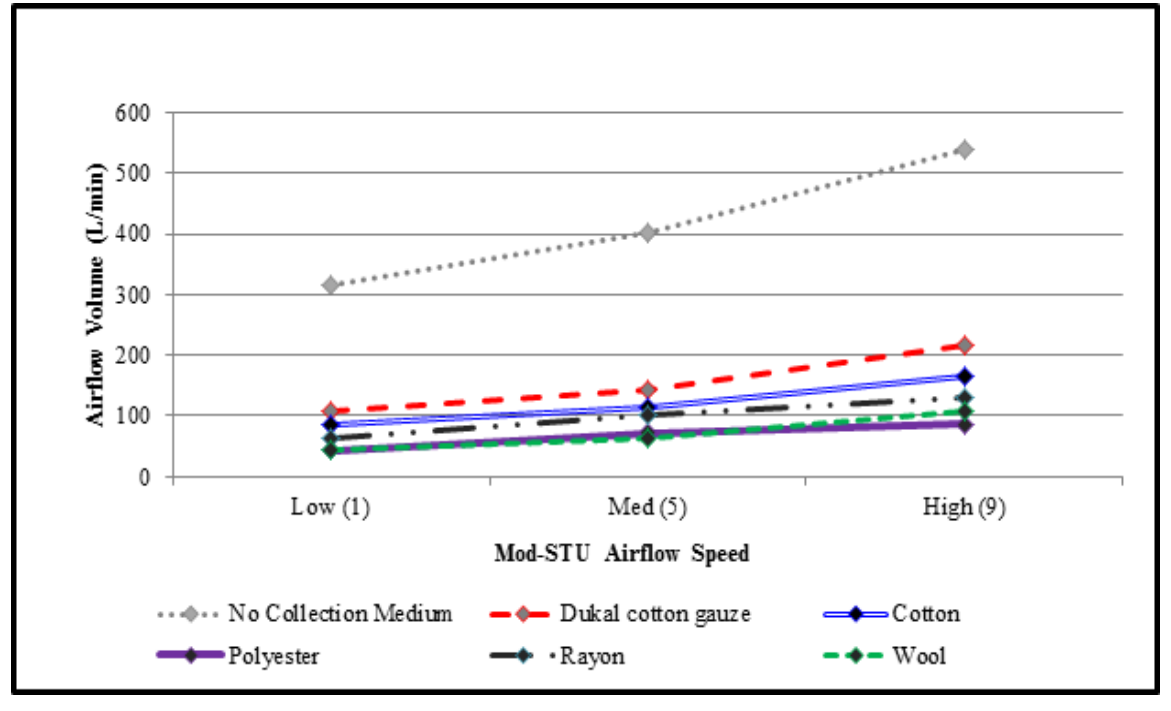

Figure 5: Airflow Volume Measurements across Sorbent Materials

Studies were conducted to evaluate this modified STU device, sampling at the low, medium and high airflow settings above the same volatile organic mix used for the direct spike study. Petri dishes were used as the volatile organic mix containment so as to provide a bigger surface area over which to sample with the device. A set of triplicate samples were conducted per airflow speed for each material tested. As can be seen in Figure 6, cotton fabric had the highest reported recovered mass at the lowest airflow setting of the device with an average of $195.04 \mathrm{ng}$. The rayon and wool fabrics also had the highest mass amounts at the low airflow speed. However, both the polyester and Dukal did not exhibit the same pattern and obtained higher mass amount at both the medium and high flows, respectively. However, it can be noted that in general the lowest airflow provide with the optimal setting considering the amount of scent mass being detected and the smaller standard error per triplicate sampling, thus indicating more reproducibility. Using a 2-way ANOVA analysis, it was shown that both fabric type and airflow speed had a statistically significant difference $(\mathrm{P}<0.001)$ in the amount of chemicals collected. Furthermore, there was a statistical significant interaction between both of these variables. The low scent mass amounts observed at the high airflow speed could be a direct result of breakthrough effects during sampling. The higher the air suction, the higher the rate compounds can be drawn past the collection material without being deposited in the fabric substrate. Even though the gauze material had the most open weave pattern, the scent mass amount at the high flow seem to be higher when compared to the other rates. This result, however, could be masking the breakthrough effect as it has the highest standard error of all sampling conducted ( $\mathrm{SE} \pm 34.6 \mathrm{ng}$ ), meaning a fairly unreproducible odor profile. 
Table 2 lists the scent mass amounts of each target human odor volatile recovered at each particular fabric/airflow speed combination. Three of the selected compounds, namely 2-furancarboxaldehyde, 2-furanmethanol and 6-methyl-5-hepten-2-one were not detected in any of the materials at none of the airflow speeds studied. Octanoic acid was only detected at the low airflow with both cotton and polyester fabric types. Phenol was never detected in the polyester fabric; however, it did have its highest reported mass in the cotton fabric. The amounts reported for each compound vary across the materials and it seems that the only general pattern observed is the higher scent mass amounts obtained with the cotton fabric at the lowest airflow speed. The low amount observed with the polyester fabric can be attributed to both chemical and morphological traits of the fiber including lower polarity, round cross-sectional shape, smooth surfaces and fewer voids of the fiber structure which hinder the retention of the chemical. The capability of collection via an airflow method is reduced due to breakthrough effects and the retention of the chemicals is lower when compared to a direct contact approach, which is on par with results using live human objects via these two approaches [23]. Nevertheless, the dynamic airflow is a viable method for collection of volatiles as observed from the optimal capability of cotton at the low airflow rate.

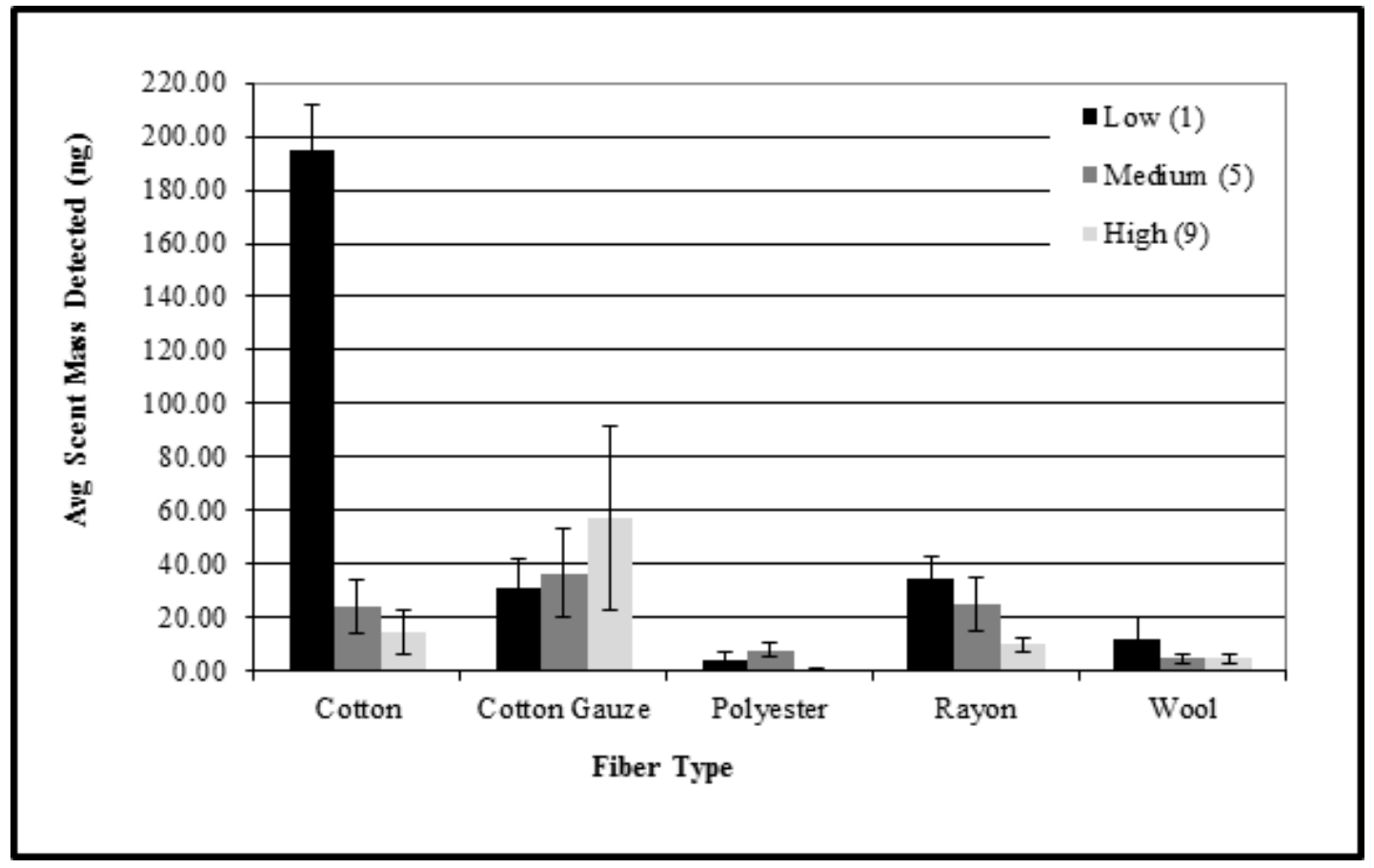

Figure 6: Total amount of standard compounds trapped and released from various fabric substrates using a dynamic airflow collection method

\section{Conclusion}

This work was performed to evaluate a range of textile substrates on the retention and subsequent release of volatiles compounds as associated with human odor. A set of target compounds were investigated via a direct spiking method as well as through the help of an airflow collection device. The direct spike experiment showed how natural, cellulosic fibers such as viscose rayon showed an enhanced ability to release a reproducible volatile odor profile. Rayon demonstrated to be the fabric type with the highest recovered scent mass amounts, followed by wool and polyester. As expected, cotton showed the lowest recovered amounts, possibly due to its complex fiber morphology, which enhances the possibility of chemicals to be retained in higher rates within the structure of the cotton fiber. Samples collected on the same fabric substrate showed a reproducible odor profile as measured via hierarchical clustering, which corroborates previous live human odor studies and which can be pivotal in forensic biometric measurements. The introduction of an airflow variable to volatile collection decreased the amounts recovered for all fiber types. The reproducibility for each fabric type between replicate sampling was also reduced and a statistical significant difference $(\mathrm{P}<0.001)$ was observed in the interaction between airflow speed and fabric type. The cotton fabric was the material which showed enhance collection at the low airflow speed as observed by the recovered mass amount. In conclusion, these findings do indicate that chemical retention is strongly affected by fiber type and outside environmental variables such as airflow. This should be taken into account in forensic cases where the use of a homogeneous fabric type must be implemented for an investigation. The use of blended fiber types may prove to be useful in a given fabric as the combination of morphological and physicochemical properties could enhance the collection, retention and release of a wider range of compound classes.

\section{Acknowledgements}

The authors would like to acknowledge the Technical Support Working Group (TSWG) for funding this research. 
Table 2: Dynamic Airflow Scent Mass Amounts (ng) Using an Array of Fabric Types

\begin{tabular}{|c|c|c|c|c|c|}
\hline & \multicolumn{5}{|c|}{ Airflow Spd 1} \\
\hline Compound & Cotton Gauze & Cotton Fabric & Polyester & Rayon & Wool \\
\hline 2-Furancarboxaldehyde & ND & ND & ND & ND & ND \\
\hline 2-Furanmethanol & ND & ND & ND & ND & ND \\
\hline Phenol & 1.4 & 4.9 & ND & 0.4 & 1.0 \\
\hline 5-Hepten-2-one, 6-methyl- & ND & ND & ND & ND & ND \\
\hline Nonanal & 3.3 & 28.7 & 0.2 & 2.5 & 1.2 \\
\hline Octanoic acid, methyl ester & 10.9 & 49.5 & ND & 2.2 & 0.8 \\
\hline Dodecane & 4.4 & 2.9 & 0.2 & ND & 2.2 \\
\hline Decanal & 2.1 & 31.7 & 0.1 & 3.9 & 0.4 \\
\hline $\begin{array}{l}\text { Hexanedioic acid, dimethyl } \\
\text { ester }\end{array}$ & 2.6 & 30.3 & ND & 11.8 & ND \\
\hline Tetradecane & 5.0 & 17.7 & 3.1 & 5.3 & 5.0 \\
\hline $\begin{array}{l}\text { 5,9-Undecadien-2-one, } \\
\text { 6,10-dimethyl }\end{array}$ & 1.4 & 25.0 & 0.4 & 8.5 & 1.7 \\
\hline \multirow[t]{2}{*}{ Octanoic acid } & ND & 4.3 & 0.2 & ND & ND \\
\hline & \multicolumn{5}{|c|}{ Airflow Spd 5} \\
\hline Compound & Cotton Gauze & Cotton Fabric & Polyester & Rayon & Wool \\
\hline 2-Furancarboxaldehyde & $\mathrm{ND}$ & ND & $\mathrm{ND}$ & ND & ND \\
\hline 2-Furanmethanol & ND & ND & ND & ND & ND \\
\hline Phenol & 0.6 & 2.5 & ND & 3.2 & ND \\
\hline 5-Hepten-2-one, 6-methyl- & ND & ND & ND & ND & ND \\
\hline Nonanal & 5.7 & 1.3 & 0.2 & 0.6 & 0.1 \\
\hline Octanoic acid, methyl ester & 10.3 & 5.3 & ND & 0.8 & ND \\
\hline Dodecane & 3.7 & 0.5 & 0.6 & ND & 0.9 \\
\hline Decanal & 8.3 & 1.4 & 0.3 & 1.3 & 0.5 \\
\hline $\begin{array}{l}\text { Hexanedioic acid, dimethyl } \\
\text { ester }\end{array}$ & 1.0 & 2.6 & ND & 5.0 & ND \\
\hline Tetradecane & 6.3 & 5.9 & 6.4 & 2.6 & 2.0 \\
\hline $\begin{array}{l}\text { 5,9-Undecadien-2-one, } \\
\text { 6,10-dimethyl }\end{array}$ & 0.8 & 4.5 & 0.4 & 11.7 & 1.0 \\
\hline \multirow[t]{2}{*}{ Octanoic acid } & ND & ND & ND & ND & ND \\
\hline & \multicolumn{5}{|c|}{ Airflow Spd 9} \\
\hline Compound & Cotton Gauze & Cotton Fabric & Polyester & Rayon & Wool \\
\hline 2-Furancarboxaldehyde & $\mathrm{ND}$ & ND & $\mathrm{ND}$ & ND & ND \\
\hline 2-Furanmethanol & $\mathrm{ND}$ & ND & ND & ND & ND \\
\hline Phenol & 2.7 & 2.7 & ND & ND & 0.1 \\
\hline 5-Hepten-2-one, 6-methyl- & ND & ND & ND & ND & ND \\
\hline Nonanal & 8.9 & 1.6 & 0.2 & 1.4 & 0.9 \\
\hline Octanoic acid, methyl ester & 6.5 & 1.7 & $\mathrm{ND}$ & 0.6 & 0.1 \\
\hline Dodecane & 2.0 & ND & ND & ND & 0.6 \\
\hline Decanal & 29.1 & 1.6 & ND & 0.9 & 0.7 \\
\hline $\begin{array}{l}\text { Hexanedioic acid, dimethyl } \\
\text { ester }\end{array}$ & 2.1 & 1.7 & ND & ND & ND \\
\hline Tetradecane & 4.0 & 3.0 & 0.7 & 1.5 & 2.0 \\
\hline $\begin{array}{l}\text { 5,9-Undecadien-2-one, } \\
\text { 6,10-dimethyl }\end{array}$ & 1.8 & 2.1 & ND & 5.4 & 0.4 \\
\hline Octanoic acid & ND & ND & ND & ND & ND \\
\hline
\end{tabular}

${ }^{\star} \mathrm{ND}=$ Not Detected

\section{References}

1. Syrotuck, William G (2000) Scent and the Scenting Dog. Mechanicsburg, PA: Barkleigh Productions, Inc.

2. Romero-Steiner S, Witek T, Balish E (1990) Adherence of Skin Bacteria to Human Epithelial Cells. J Clin Microbiol 28: 27-31.

3. Leyden JJ, McGinley KJ, Hölzle E, Labows JN, Kligman AM (1981) The microbiology of the human axilla and its relationship to axillary odor. J Invest Dermatol 77: 413-6.

4. R R Roth, W D James (1988) Microbial Ecology of the Skin. Annual Review of Microbiology 42: 441-64.

5. Teufel L, Schuster KC, Merschak P, Bechtold T, Redl B (2007) Development of a fast and reliable method for the assessment of microbial colonization and growth on textiles by DNA quantification. J Mol Microbiol Biotechnol 14: 193-200.

6. JMM.Malheiroa, LRS Salvadoa (2009) Microscopic study of bacteria-textile material interaction for hygienic purpose. Microscopy and Microanalysis 15: 63-64. 
7. Hsieh YL, Merry J (1986) The adherence of Staphylococcus aureus, Staphylococcus epidermidis and Escherichia coli on cotton, polyester and their blends. J Appl Bacteriol 60: 535-44.

8. Wilkoff LJ, Westbrook L, Dixon GJ (1969) Factors affecting the persistence of Staphylococcus aureus on fabrics. Appl Microbiol 117: 268-74.

9. Hsieh YL, Timm DA, Merry J (1987) Bacterial Adherence on Fabrics by a Radioisotope Labeling Method. Textile Research Journal 57: 20-28.

10. Sidwell RW, Dixon GJ, McNeil E (1966) Quantitative studies on fabrics as disseminators of viruses. I. Persistence of vaccinia virus on cotton and wool fabrics. Appl Microbiol 14: 55-9.

11. Balseiro SC, Correia HR (2006) Is olfactory detection of human cancer by dogs based on major histocompatibility complex-dependent odour components? A possible cure and a precocious diagnosis of cancer. Med Hypotheses 66: 270-2.

12. Ostrovskaya A, Landa PA, Sokolinsky M, Rosalia AD, Maes D (2001) Study and identification of volatile compounds from human skin. Journal of Cosmetic Science 53: 147-148.

13. Bernier UR, Kline DL, Barnard DR, Schreck CE, Yost RA (2000) Analysis of human skin emanations by gas chromatography/mass spectrometry. 2. Identification of volatile compounds that are candidate attractants for the yellow fever mosquito (Aedes aegypti). Anal Chem 72: 747-56.

14. Braks MAH, Scholte EJ, Takken W, Dekker T (2000) Microbial growth enhances the attractiveness of human sweat for the malaria mosquito, Anopheles gambiae sensu stricto (Diptera: Culicidae). Chemoecology 10: 129-34.

15. Meijerink J, Braks MAH, Brack AA, Adam W, Dekker T, et al. (2000) Identification of Olfactory Stimulants for Anopheles gambiae from Human Sweat Samples. Journal of Chemical Ecology 26: 1367-82.

16. Logan JG, Birkett MA, Clark SJ, Powers S, Seal NJ, et al. (2008) Identification of human-derived volatile chemicals that interfere with attraction of Aedes aegypti mosquitoes. J Chem Ecol 34: 308-22.

17. Schoon GAA, Curran AM, Furton KG (2009) Odours and biometrics. In: Li SZ, editor. Encyclopedia of biometrics. Secaucus, NJ: Springer $1003-1008$.

18. Obendorf SK, Liu H, Leonard MJ, Young TJ, Incorvia MJ (2005) Effects of aroma chemical vapor pressure and fiber morphology on the retention of aroma chemicals on cotton and poly (ethylene terephthalate) fabrics. Journal of Applied Polymer Science 99: 1720-23.

19. Liu H, Obendorf SK, Young TJ, Incorvia MJ (2004) Microscopic analysis of aroma chemical distribution on fibers. I. cis-3-hexenyl salicylate. Journal of Applied Polymer Science 91: 3557-64.

20. Liu H, Obendorf SK, Leonard MJ, Young TJ, Incorvia MJ (2005) Adsorption of aroma chemicals on cotton fabric from aqueous systems. Journal of surfactants and detergents 8: 311-17.

21. Curran AM, Ramirez CF, Schoon AA, Furton KG (2007) The frequency of occurrence and discriminatory power of compounds found in human scent across a population determined by SPME-GC/MS. Journal of Chromatography B 846: 86-97.

22. Curran AM, Prada PA, Furton KG (2009) The Differentiation of the Volatile Organic Signatures of Individuals Through SPME-GC/MS of Characteristic Human Scent Compounds. Journal of Forensic Sciences 55: 50-7.

23. Prada PA, Curran AM, Furton KG (2011) The evaluation of human hand odor volatiles on various textiles: a comparison between contact and noncontact sampling methods. J Forensic Sci 56: 866-81.

24. Hudson-Holness DT, Furton KG (2010) Comparison between Human Scent Compounds Collected on Cotton and Cotton Blend Materials for SPME-GC/MS Analysis. Journal of Forensic Research doi:10.4172/2157-7145.1000101.

25. DeGreeff LE, Curran AM, Furton KG (2011) Evaluation of selected sorbent materials for the collection of volatile organic compounds related to human scent using non-contact sampling mode. Forensic Science International 209: 133-42.

26. Prada PA, Curran AM, Furton KG (2010) Comparison of extraction methods for the removal of volatile organic compounds (VOCs) present in sorbents used for human scent evidence collection. Anal Methods 2: 470-8.

27. Fletcher K (2007) Optimization and Development of a Human Scent Collection Method. Thesis. Bethsada, Maryland: Uniformed Services University of the Health Sciences. 